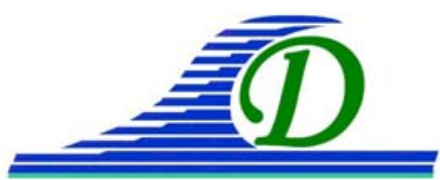

XIII $^{\text {èmes }}$ Journées Nationales Génie Côtier-Génie Civil Dunkerque, 2-4 juillet 2014

DOI:10.5150/jngcgc.2014.017_ C Editions Paralia CFL disponible en ligne - $h$ ttp://www.paralia.fr - available online

\title{
Implémentation d'un nouveau modèle opérationnel de prévision des vagues et surcotes marines
}

\section{Audrey PASQUET ${ }^{1}$, Héloïse MICHAUD ${ }^{1}$, Lotfi AOUF $^{2}$, Rémy BARAILLE $^{1}$, Caroline BRU ${ }^{1}$, Stéphanie CORREARD ${ }^{1}$, Flavien GOUILLON ${ }^{1}$, Didier JOURDAN $^{1}$, Guillaume MORVAN ${ }^{2}$, Patrick OHL ${ }^{2}$, Denis PARADIS ${ }^{2}$}

1. Service hydrographique et océanographique de la Marine,

Météo-France, Bâtiment Laplace, 42 avenue G. Coriolis, BP 63576-31035, Toulouse Cedex, France.

audrey.pasquet@shom.fr ; heloise.michaud@shom.fr

2. Météo-France, département de la prévision marine,

Bâtiment Poincaré, 42 avenue Coriolis, 31100 Toulouse, France.

\section{Résumé :}

Les modèles HYCOM et Wavewatch III seront implémentés dans la chaîne opérationnelle de Vigilance Vague Submersion de Météo-France d'ici 2015 (projet HOMONIM). Dans ce papier sont présentés une évaluation des performances de ces modèles sur des rejeux de tempêtes, et les résultats d'un premier couplage via la prise en compte de la rugosité de surface dans le modèle de surcote et l'effet des courants et variation des niveaux dans le modèle de vagues.

Mots-clés : Océanographie opérationnelle côtière, Surcote, Vagues, HYCOM, Wavewatch III, HOMONIM.

\section{Introduction}

La modélisation des surcotes marines et des principaux processus physiques affectant les zones côtières requiert l'utilisation de modèles de circulation et de vagues à de hautes résolutions spatiales et temporelles. Ceci est possible par l'utilisation de techniques d'emboîtement, ou par l'implémentation de grilles à résolution variable, de type curvilinéaires ou non-structurées. Dans le cadre du volet modélisation du projet HOMONIM (Historique, Observation, MOdélisation des NIveaux Marins, projet commun au SHOM et Météo-France, 2012-2015), la seconde approche a été retenue, afin de développer une capacité opérationnelle de prévision des hauteurs d'eau, des surcotes et des états de mer à la côte qui améliore la pertinence de la Vigilance VaguesSubmersion (VVS) déjà existante. Ce projet, visant à améliorer la connaissance et la prévision de l'aléa marin, est sous maîtrise d'ouvrage de la Direction Générale pour la Prévention des Risques (DGPR), et s'inscrit dans le Plan interministériel Submersions Rapides. Il fait suite aux événements tempétueux du type Xynthia, et profite d'une importante augmentation des capacités de calcul opérationnelles avec l'installation du nouveau supercalculateur de Météo-France, qui permet désormais l'utilisation de 
maillages très raffinés dans les modèles de prévision. Le modèle de circulation HYCOM (BLECK, 2002) remplace d'ores et déjà le modèle de Météo-France depuis Janvier 2014. Le modèle Wavewatch III @ (WW3, TOLMAN 2008) sera opérationnel début 2015 et complètera, en zone côtière, le modèle MFWAM (LEFEVRE et al., 2009) déjà utilisé en zone hauturière.

Les modèles HYCOM et WW3 et leurs paramétrisations sont décrits en section 2. Les performances des deux modèles sont présentées en section 3.1. Une première évaluation de l'apport d'un couplage entre modèles est présentée section 3.2, avec la prise en compte des états de mer dans la tension de surface du modèle de surcote, et des niveaux d'eau et courants dans le modèle de vagues. Enfin, des pistes d'amélioration du dispositif global sont discutées dans la conclusion.

\section{Modèles et configurations}

\subsection{HYCOM}

Pour le calcul des hauteurs d'eau, on utilise le modèle HYCOM (version du SHOM, BARAILLE \& FILATOFF, 1995) en configuration barotrope. La dynamique est modélisée sur deux domaines, l'un dit "ATL" couvrant $43^{\circ} \mathrm{N}$ à $62^{\circ} \mathrm{N}$ et $9^{\circ} \mathrm{W}$ à $10^{\circ} \mathrm{E}$, et l'autre dit "MED" couvrant $30^{\circ} \mathrm{N}$ à $46^{\circ} \mathrm{N}$ et $9^{\circ} \mathrm{W}$ à $37^{\circ} \mathrm{E}$.

HYCOM utilisait jusqu'en 2012 des grilles uniformes. Le code a été adapté aux grilles curvilinéaires afin d'optimiser le rapport résolution/performance du modèle pour les besoins opérationnels. Une méthode de projection bipolaire de type Mercator a permis de construire deux maillages curvilinéaires de résolution élevée aux côtes françaises, de l'ordre du kilomètre voire jusqu'à $400 \mathrm{~m}$ dans le Golfe Normand Breton, et plus lâche dans l'océan hauturier. La grille ATL non reprojetée est illustrée figure 1.

Des études paramétriques sur les conditions aux limites (forçages de marée, type de conditions aux limites), l'emprise et la résolution du maillage, et la friction de fond, ont permis de valider la marée modèle par rapport aux prédictions de marée du SHOM. Une bonne représentation de la marée est critique dans les zones de forts courants et de faible profondeur comme la Manche, où il existe une interaction non négligeable entre marée et surcote via la friction de fond (IDIER et al., 2012). Nos études ont montré que ces interactions sont en revanche négligeables en Méditerranée Occidentale. Dans ce domaine, la représentation des effets de bassin requiert une résolution au moins kilométrique au niveau du détroit de Gibraltar, ainsi qu'une emprise couvrant l'ensemble des bassins orientaux et occidentaux.

Le modèle utilise la bathymétrie fournie par le LEGOS (Laboratoire d'Etudes en Géophysique et Océanographie Spatiales, Toulouse) et est forcé en élévation de surface par les 15 composantes de l'atlas de marée NEA Optimal 2011 (données fournies par F. Lyard du LEGOS). L'utilisation de bathymétries plus résolues à la côte est un axe 


\section{XIII ${ }^{\text {èmes }}$ Journées Nationales Génie Côtier - Génie Civil \\ Dunkerque, 2-4 juillet 2014}

d'amélioration du système de prévision et fera l'objet de tests d'ici fin 2014 (BISCARA et al., 2014).

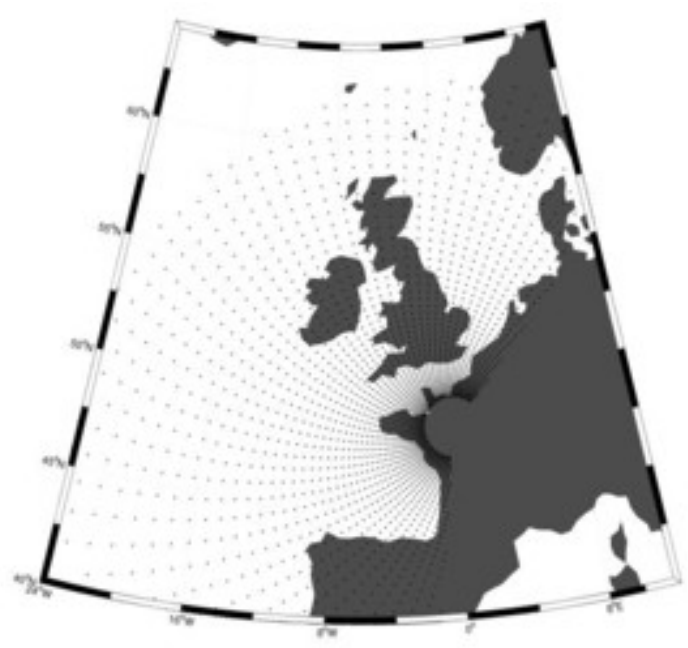

Figure 1. Maillage curvilinéaire de la maquette ATL avec augmentation de la résolution sur les côtes françaises (1 point sur 12 représenté). La localisation du pôle sud sur la France permet une haute résolution aux côtes françaises.

Les tests paramétriques à la valeur du coefficient de friction de fond $\mathrm{Cb}$, pour une paramétrisation quadratique spatialement constante, ont montré une sensibilité de la marée à la friction significative en Manche et réduite sur les façades Atlantique-Golfe de Gascogne et Méditerranéenne. Sur le domaine ATL, le Cb a donc été calculé selon une formulation logarithmique de la vitesse verticale qui tient compte de la longueur de rugosité. Il a ensuite été ajusté en Manche par une recherche de $\mathrm{Cb}$ optimal qui minimise les erreurs sur les phases et amplitudes de la marée à des points marégraphiques. La friction de fond obtenue est spatialement variable et spécifiquement adaptée à la dissipation numérique du modèle. Sur le domaine MED, la paramétrisation quadratique avec un coefficient constant à 0,002 est conservée.

A l'issue de ces tests paramétriques, deux configurations curvilignes optimales pour la modélisation de la marée ont été arrêtées en ATL et MED. Elles ont permis une amélioration des scores moyens de marée en ATL par rapport au modèle de MétéoFrance de $50 \%$ et $60 \%(\sim 10-20 \mathrm{~cm})$ sur les biais de basses et pleines mers, et de $35 \%$ ( 10 minutes) sur le déphasage (PARADIS et al., 2013).

Les forçages atmosphériques pour la modélisation des surcotes sont issus du modèle atmosphérique Arpège $1 / 4^{\circ}$ à $3 \mathrm{~h}$ (prévisions), avec un complément basse résolution Arpège au $1 / 2^{\circ}$ à $6 \mathrm{~h}$ (analyses) pour les tempêtes à partir de 2007. Les forçages pour les événements plus anciens sont issus d'une reconstitution météorologique à partir de la ré-analyse du CEP (ERA-Interim) associée à une descente d'échelle Aladin pour obtenir des champs météorologiques horaires à la résolution $1 / 10^{\circ}$. 
Les travaux de IDIER et al. (2012), ont permis de cibler, pour le calcul du stress de vent, les formulations de Wu (WU, 1985) et de Charnock (CHARNOCK, 1955) comme étant les plus optimales. Des études complémentaires plus poussées sur la paramétrisation constante de Charnock et sur la formulation de Makin (MAKIN, 2005) ont finalement mené au choix d'un coefficient $C_{d}$ pour le calcul de la tension de vent obtenu selon les équations de Charnock, Eq. 1 , où $z_{0}$ est la longueur de rugosité, $\mathrm{u}_{*}$ est la vitesse de friction, $\alpha$ est le paramètre de Charnock constant, variant dans la littérature entre 0.01 et 0.025 (MAKIN, 2005), $\kappa=0.4$ est la constante de Von Karman.

$$
C_{d}=\frac{u_{*}{ }^{2}}{u_{z}{ }^{2}}=\kappa^{2}\left[\ln \left(z / z_{0}\right)\right]^{-2}, z=10 m, z_{0}=\alpha \frac{u_{*}{ }^{2}}{g}
$$

Le paramètre empirique $\alpha$ constant spatialement représente l'effet des vagues sur la tension de surface. Un coefficient $\alpha$ élevé à 0.025 a permis de réduire de $5 \mathrm{~cm}$ l'erreur moyenne sur le pic de surcote par rapport au modèle existant utilisant une paramétrisation de Wu. La formulation de Makin, a priori plus physique, n'a pas permis d'améliorer ces résultats. La formulation de Charnock à paramètre variable est un axe d'amélioration dont les premiers travaux sont présentés dans la section 3.2.1.

Un processus d'intercomparaison entre les résultats des modèles HYCOM, TELEMAC (équipe du Laboratoire National d'Hydraulique et Environnement, Chatou) et l'existant dans la VVS (équipe de Météo-France) a montré des performances globalement meilleures pour HYCOM en ATL, et équivalentes en MED entre les trois modèles.

\subsection{Wavewatch III}

Le modèle de génération et propagation des vagues MFWAM de Météo-France est utilisé actuellement pour les prévisions opérationnelles des états de mer à l'échelle régionale sur l'Atlantique Nord-Est et la Méditerranée occidentale avec une résolution de grille de $0,1^{\circ}$. Il est alors complété en zone côtière et littorale par le modèle WW3, qu'il force aux frontières ouvertes au large. Les récents développements de WW3 (version 4.18) portant sur l'ajout de paramétrisations en eaux peu profondes sur le déferlement ou la friction sur le fond (ARDHUIN et al., 2010) ont permis d'étendre sa validité aux zones côtières et littorales. Deux maillages non structurés allant de $10 \mathrm{~km}$ de résolution au large jusqu'à $200 \mathrm{~m}$ à la côte ont été implémentés sur les façades françaises méditerranéenne et atlantique.

Pour l'Atlantique, un maillage non structuré a été réalisé à partir du maillage NorgasUG décrit dans BOUDIERE et al. (2013) et utilisé pour Prévimer. Ce maillage a ensuite été tronqué afin de limiter son extension et ne couvrir que les zones d'emprise du modèle atmosphérique Arome de Météo-France (figure 2). Le maillage est composé de 93787 nœuds, il rassemble différents polygones ayant des critères de raffinement différents sur la bathymétrie et le critère CFL. Un maillage non structuré en Méditerranée de 92667 nœuds a été réalisé dans le cadre de ce projet, en utilisant le 


\section{XIII ${ }^{\text {èmes }}$ Journées Nationales Génie Côtier - Génie Civil \\ Dunkerque, 2-4 juillet 2014}

mailleur Polymesh développé par A. Roland (T.U. Darmstadt). Il est constitué de polygones dans lesquels des critères de raffinement différents autour de la Corse, du littoral français et du littoral italien ont été prescrits. Des bathymétries ont été réalisées à partir de mesures du SHOM et de l'Ifremer (MNT de façade de $100 \mathrm{~m}$ de résolution complété par la bathymétrie Emodnet au large) (BISCARA et al., 2014). Les paramétrisations physiques correspondant au TEST 451 (ARDHUIN et al., 2010) ont été utilisées en Atlantique, et au TEST 405 en Méditerranée. Pour les deux configurations, le spectre des vagues est discrétisé sur 24 directions et sur 30 fréquences entre $0.0345 \mathrm{~Hz}$ et $0.547 \mathrm{~Hz}$.

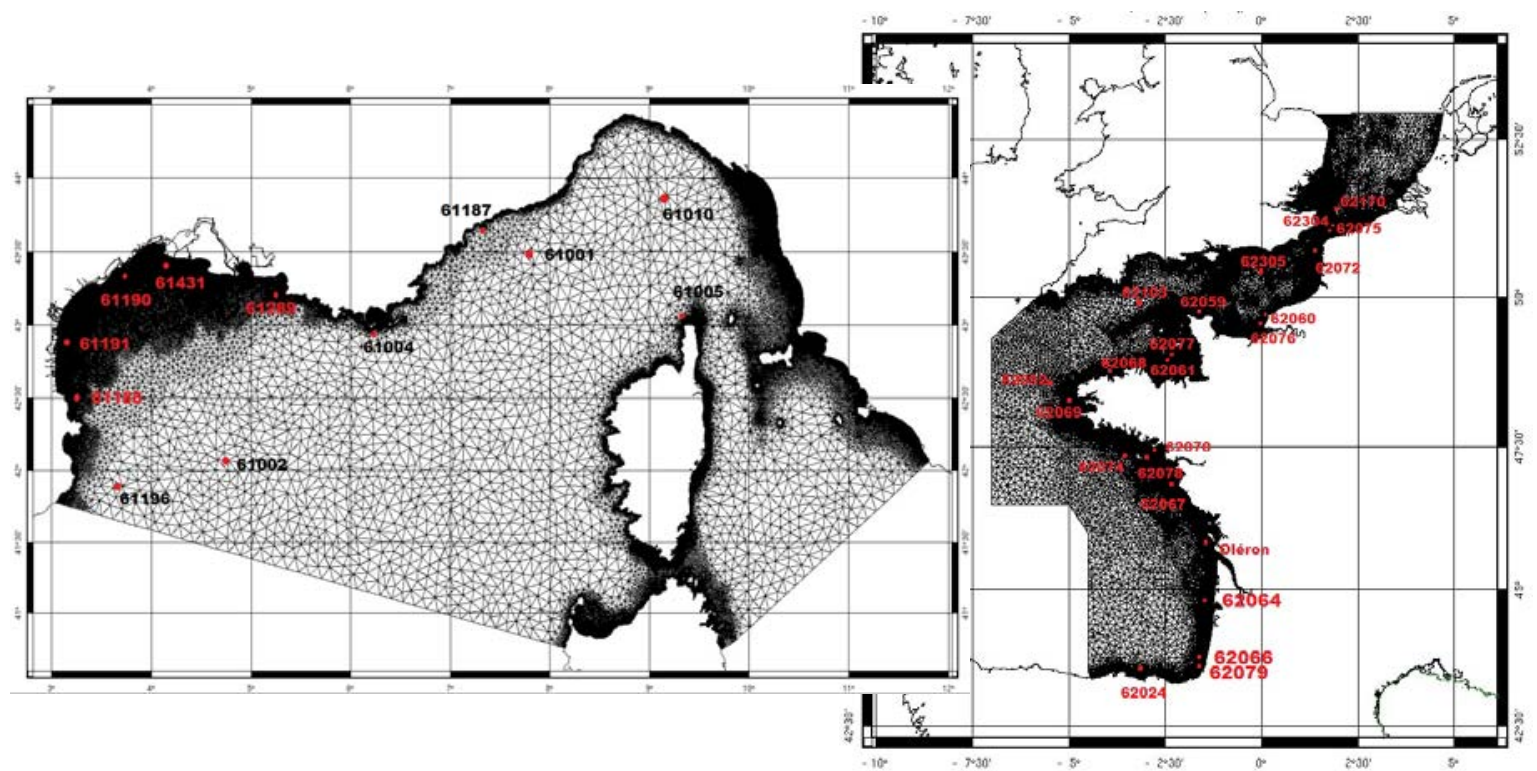

Figure 2. Maillages en Méditerranée (gauche) et en Atlantique (droite) réalisés pour le modèle de vagues. Les bouées sont représentées par des points rouges.

\section{Performances des systèmes actuels et tests vers un premier couplage}

\subsection{Résultats du dispositif actuel}

Pour chaque façade, les configurations optimales pour les deux modèles ont été évaluées à partir de simulations de 11 tempêtes significatives sélectionnées par MétéoFrance entre 1998 et 2010 et sur un rejeu d'un an (entre mi-2011 et mi-2012).

\subsubsection{Modèle de surcote}

Les performances du modèle HYCOM sur le domaine ATL dans sa configuration de référence avec un coefficient de Charnock $\alpha$ à 0.025 sont rassemblées tableau 1. Les scores statistiques ont été calculés à partir de comparaisons aux données du réseau marégraphique RONIM pour les sites de surcote significative (PARADIS et al., 2013). Les statistiques moyennées sur les 11 événements montrent une sous estimation sur le pic de surcote de l'ordre de $10 \mathrm{~cm}(7 \mathrm{~cm}$ sur la hauteur totale) et un déphasage sur 


\section{Thème 1 - Hydrodynamique côtière}

hauteur totale de 12 minutes. Les simulations longues donnent une estimation plus générale du comportement du modèle, avec un biais moyen négatif de $-2 \mathrm{~cm}$. Le biais négatif plus marqué à $-5 \mathrm{~cm}$ sur les populations des plus fortes surcotes confirme la légère sous-estimation observée sur les cas de tempêtes.

Tableau 1. Scores de la configuration ATL. Statistiques moyennées sur les simulations courtes et sur la simulation annuelle, avec et sans seuillage de la surcote dans le calcul.

\begin{tabular}{llllllllllll}
\hline $\begin{array}{l}\text { Données en cm ou } \\
\text { minutes }\end{array}$ & $\begin{array}{l}\text { Biais } \\
\text { (VA) }\end{array}$ & Biais & $\begin{array}{l}\text { Erreur } \\
\text { quad. }\end{array}$ & $\begin{array}{l}\text { Ecart } \\
\text { type }\end{array}$ & $\begin{array}{l}\text { Erreur } \\
\text { Max. }\end{array}$ & $\begin{array}{l}\text { Erreur } \\
\text { Pic }\end{array}$ & $\begin{array}{l}\text { Erreur } \\
\text { Pic }\end{array}$ & Deph. Erreur & Erreur & Deph. \\
& & & & & & (VA) & & & (VA) & Htot Pic & Htot \\
\hline Evénements & 9.3 & -4.9 & 18.7 & 18.1 & 35.5 & 16.6 & -10.4 & 46.2 & 15.3 & -7.7 & 12.2 \\
Annuel (sans seuil) & 3.8 & -2.1 & 7.9 & 7.6 & & & & & & & \\
Annuel (avec seuil) & 7 & -4.6 & 14.4 & 13.6 & & & & & & \\
\hline
\end{tabular}

Les prévisions de surcote des derniers événements de tempêtes de l'hiver 2013-2014, ont montré une tendance d'HYCOM à légèrement surestimer le pic de surcote. Ces écarts qui subsistent entre modèle et observations peuvent résulter :

- de mécanismes 3D non résolus dans le modèle,

- de la sensibilité du modèle aux forçages atmosphériques (le choix de ces derniers étant dicté par les contraintes opérationnelles, leur influence n’a pas été étudiée, mais elle peut dépasser $10 \%$ de la surcote),

- d'une friction de fond insuffisamment adaptée ; nos derniers travaux utilisant une approche stochastique pour l'approximation de la friction de fond ont permis de réduire l'erreur quadratique sur les pleines et basses mers de $12 \%$ soit $\sim 8 \mathrm{~cm}$,

- des états de mer non considérés dans le modèle de courant (composante de l'ordre de 30\% de la surcote lors de la tempête Xynthia selon BERTIN et al., 2012). Ce dernier axe d'amélioration fait l'objet des premiers résultats présentés section 3.2.

\subsubsection{Modèle de vague}

Grâce à des comparaisons à des mesures de bouées houlographes du CEREMA et Météo-France, ainsi que des mesures altimétriques issues des données du CERSAT, les performances des configurations ont pu être calculées. Des tests de sensibilité sur la résolution du maillage, les forçages atmosphériques et la friction de fond ont permis de retenir pour configuration optimale notre simulation de référence. Pour l'Atlantique, nous obtenons de bons résultats avec le modèle atmosphérique Arome (tableau 2) mais également avec d'autres modèles atmosphériques. Les coefficients de corrélation sont compris entre 84 et 98\%, avec un biais assez faible et des scatters-index (SI) et des erreurs quadratiques moyennes (RMSE) assez bons. Les résultats sont aussi comparés aux traces altimétriques des satellites Envisat, Jason 1 et 2 (tableau 3). 


\section{XIII ${ }^{\text {èmes }}$ Journées Nationales Génie Côtier - Génie Civil \\ Dunkerque, 2-4 juillet 2014}

Tableau 2. Comparaison statistique des hauteurs significatives entre le modèle forcé par Arome et les mesures pour la simulation longue.

\begin{tabular}{lllllllllllll}
\hline Bouée & $\mathbf{6 2 0 5 9}$ & $\mathbf{6 2 0 6 4}$ & $\mathbf{6 2 0 6 6}$ & $\mathbf{6 2 0 6 7}$ & $\mathbf{6 2 0 6 8}$ & $\mathbf{6 2 0 6 9}$ & $\mathbf{6 2 0 7 2}$ & $\mathbf{6 2 0 7 4}$ & $\mathbf{6 2 0 7 6}$ & $\mathbf{6 2 0 7 7}$ & $\mathbf{6 2 0 7 8}$ \\
\hline Biais (\%) & 0.01 & 0.02 & -0.02 & 0.11 & 0.35 & 0.09 & 0.04 & 0.05 & 0.03 & 0.06 & 0.07 \\
Corr. & 0.95 & 0.97 & 0.96 & 0.96 & 0.84 & 0.96 & 0.96 & 0.97 & 0.95 & 0.96 & 0.98 \\
RMSE & 0.09 & 0.15 & 0.17 & 0.23 & 0.12 & 0.38 & 0.11 & 0.15 & 0.10 & 0.10 & 0.13 \\
S.I. & 0.16 & 0.13 & 0.15 & 0.17 & 0.46 & 0.17 & 0.17 & 0.13 & 0.19 & 0.14 & 0.15 \\
\hline
\end{tabular}

Tableau 3. Comparaison statistique des hauteurs significatives entre le modèle forcé par Arome et les mesures altimétriques pour la simulation longue.

\begin{tabular}{llll}
\hline Satellite & Biais & RMSE & SI \\
\hline Envisat & 0.067 & 0.263 & 0.137 \\
Jason 1 & 0.076 & 0.273 & 0.144 \\
Jason 2 & 0.043 & 0.274 & 0.138 \\
\hline
\end{tabular}

\subsection{Vers un couplage entre les modèles}

\subsubsection{Prise en compte des états de mer dans HYCOM}

Les tempêtes de 2007 à 2010 ont été rejouées pour des coefficients de Charnock $\alpha$ variant de 0.01 à 0.025 (expérience de référence), ainsi qu'avec un coefficient de Charnock variable calculé par le modèle de vagues WW3 sur une grille régionale de 10 $\mathrm{km}$ de résolution. La population statistique considérée est de 29 données (couple [tempête, site marégraphique]). L'objectif de cette étude préliminaire est moins de démontrer l'apport du coefficient variable, ce qui nécessitera une population statistique plus fournie représentative des différents types de tempêtes, que d'évaluer l'effet d'un coefficient constant $\alpha$ plus physique se rapprochant du coefficient variable. Les scores moyennés sur les sites de surcote significative et les 5 tempêtes figurent dans le tableau 4.

Tableau 4. Scores de la configuration ATL pour $\alpha$ entre 0,01 et 0,025, et prenant en compte les états de mer (Charnock variable). Statistiques moyennées sur 5 tempêtes et sur les sites marégraphiques d'intérêt.

\begin{tabular}{|c|c|c|c|c|c|c|c|c|c|c|c|}
\hline $\begin{array}{l}\text { Données en } \mathrm{cm} \\
\text { ou minutes }\end{array}$ & $\begin{array}{l}\text { Biais } \\
\text { (VA) }\end{array}$ & Biais & $\begin{array}{l}\text { Erreur } \\
\text { quad. } \\
\text { moyenne }\end{array}$ & $\begin{array}{l}\text { Ecart } \\
\text { type }\end{array}$ & $\begin{array}{l}\text { Erreur } \\
\text { Maximale }\end{array}$ & $\begin{array}{l}\text { Erreur } \\
\text { Pic (VA) }\end{array}$ & $\begin{array}{l}\text { Erreur } \\
\text { Pic }\end{array}$ & Deph. & $\begin{array}{l}\text { Erreur } \\
\text { Pic Htot } \\
\text { (VA) }\end{array}$ & $\begin{array}{l}\text { Erreur } \\
\text { Pic Htot }\end{array}$ & $\begin{array}{l}\text { Deph } \\
\text { Htot }\end{array}$ \\
\hline Charnock0.010 & 12.2 & -11.1 & 18.8 & 12.4 & 37.9 & 28.4 & -28.4 & -5.5 & 16.4 & -15.4 & 2.8 \\
\hline Charnock0.013 & 11.1 & -9.6 & 18.0 & 12.2 & 36.9 & 25.0 & -25.0 & -3.4 & 14.5 & -13.3 & 2.4 \\
\hline Charnock0.016 & 10.4 & -8.3 & 17.3 & 12.1 & 36.1 & 22.0 & -22.0 & -1.7 & 13.0 & -11.4 & 1.0 \\
\hline Charnock0.019 & 9.9 & -7.4 & 16.9 & 12.0 & 35.5 & 19.3 & -19.3 & -2.1 & 12.1 & -9.7 & 2.4 \\
\hline Charnock0.022 & 9.5 & -6.3 & 16.6 & 12.1 & 35.4 & 17.2 & -16.9 & -3.1 & 11.5 & -8.2 & 2.4 \\
\hline Charnock0.025 & 9.1 & -5.3 & 16.4 & 12.1 & 35.2 & 15.6 & -14.5 & -2.4 & 11.6 & -6.7 & 1.7 \\
\hline Charnock-Variable & 9.6 & -6.9 & 16.8 & 12.1 & 35.7 & 18.0 & -18.0 & -2.4 & 11.9 & -9.2 & 4.1 \\
\hline
\end{tabular}


La paramétrisation de Charnock variable ne permet l'amélioration d'aucun indicateur statistique par rapport à la référence ; elle dégrade les erreurs d'amplitude et de phase au niveau du pic de surcote et intensifie la sous-estimation moyenne observée pour la configuration de référence. Les performances moyennes de la paramétrisation de Charnock variable sont similaires à une paramétrisation de Charnock constant pour $\alpha$ compris entre 0.019 et 0.022 . Les résultats par sites et tempêtes pour ces trois expériences (non illustrés) montrent des scores globalement meilleurs pour $\alpha=0.022$, mais suggèrent une erreur sur le pic de surcote réduite avec le Charnock variable pour les sites en Manche et au niveau du détroit du Pas de Calais.

\subsubsection{Prise en compte des niveaux d'eau et courants dans WW3}

Sur la période de la tempête Johanna (4 au 12/03/2008), caractérisée par une mer très forte associée à un grand coefficient de marée, un test de sensibilité à l'apport des niveaux et courants a été réalisé. Un premier test a consisté à prendre en compte les niveaux issus d'HYCOM pour cette tempête, et un second test a pris en compte les niveaux et courants barotropes. Le coefficient de corrélation des Hs a été amélioré, passant de 0.91 à 0.95 lors de la prise en compte des niveaux (tableau 5), et les tendances sont bien reproduites. En revanche, un biais légèrement accru est toujours présent.

A Cherbourg, l'Ile d'Yeu et aux Pierres-Noires, les marées ont induit des oscillations dans les hauteurs significatives, qui peuvent être (à l'Ile d'Yeu) ou non corrélées temporellement à celles observées (figure 3). On constate en outre que la prise en compte des courants a plutôt dégradé légèrement les résultats en comparaison de la simulation avec seulement la prise en compte des niveaux, excepté à Cherbourg où la hauteur des vagues qui était surestimée dans les autres simulations, s'est rapprochée de la mesure. L'utilisation de courants barotropes comme forçage dans un modèle de vague n'est cependant pas adaptée, et il faudrait plutôt utiliser les courants de surface, soit fournis par un modèle 3D, soit calculé à partir d'une analyse sur les courants barotropes. Enfin, une comparaison avec des mesures en zone peu profonde avec un fort marnage serait nécessaire pour aller plus loin.

Tableau 5. Statistiques moyennées sur les différentes bouées pour la tempête de mars 2008 de la configuration Atlantique pour Hs de la simulation de référence, celle avec les niveaux d'eau et celle avec les niveaux et courants.

\begin{tabular}{llll}
\hline & Simulation de référence & Simulation avec niveaux & $\begin{array}{l}\text { Simulation avec niveaux } \\
\text { et courant }\end{array}$ \\
\hline Biais & 0.18 & 0.24 & 0.24 \\
Corr. & 0.91 & 0.95 & 0.94 \\
RMSE & 0.58 & 0.61 & 0.64 \\
S.I. & 0.30 & 0.31 & 0.32 \\
\hline
\end{tabular}




\section{XIII ${ }^{\text {èmes }}$ Journées Nationales Génie Côtier - Génie Civil \\ Dunkerque, 2-4 juillet 2014}

\section{Pistes d'amélioration et conclusions}

Les résultats montrent que l'apport d'une paramétrisation de la tension de vent utilisant un paramètre de Charnock variable calculé par un modèle de vague régional n'est pas significatif comparé à un coefficient de Charnock constant empirique. Une étude plus avancée portera sur une simulation d'un an ainsi que sur les tempêtes de l'hiver 20132014, pour lesquelles d'importantes composantes marée et vagues conjuguées ont engendré des submersions répétées. La prise en compte des niveaux a légèrement amélioré les résultats du modèle de vagues, mais le forçage par les courants barotropes n'a pas été concluant, ce qui confirme la nécessité d'utiliser les courants de surface. De futurs travaux porteront sur un couplage direct entre les modèles HYCOM et WW3 via l'approche basée sur la théorie de la moyenne lagrangienne généralisée, glm2z-RANS (ARDHUIN et al., 2008, MICHAUD et al., 2012).
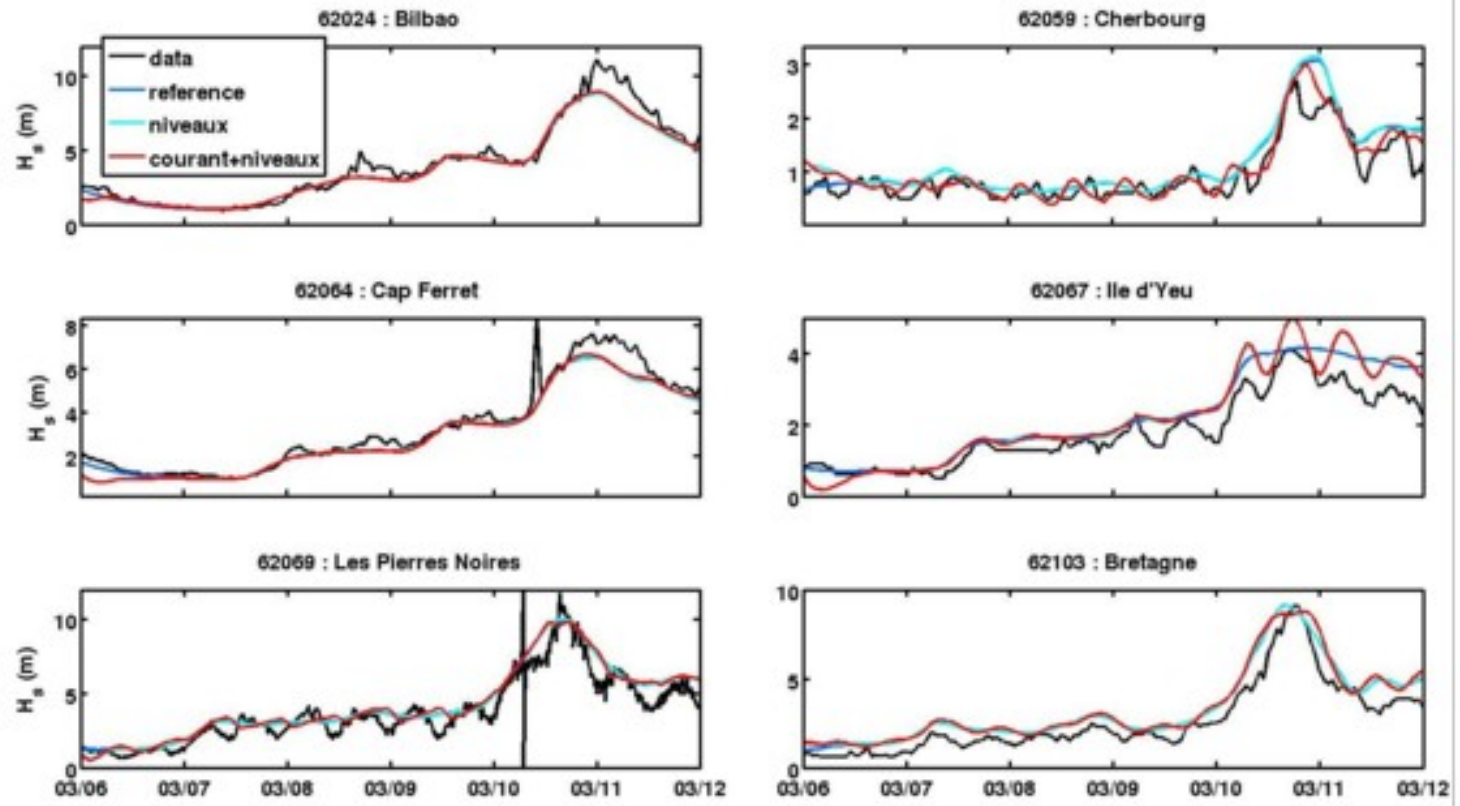

Figure 3. Séries temporelles des Hs pour la simulation de référence (bleu), celle avec les niveaux d'eau issus d'HYCOM (cyan) et celle avec les niveaux et courants issus d'HYCOM (rouge), comparées aux mesures (noir) pour la tempête de mars 2008.

\section{Remerciements}

Cette étude s'inscrit dans le projet HOMONIM financé par la DGPR. Nous remercions le Cetmef pour les mesures de vagues, le CERSAT pour les mesures altimétriques, et le LEGOS pour les forçages de marée NEA.

\section{Références bibliographiques}

ARDHUIN F., RASCLE N., BELIBASSAKIS K.A. (2008). Explicit wave-averaged primitive equations using a generalized Lagrangian mean. Ocean Modelling, Vol. 20(1), pp 35-60. http://dx.doi.org/10.1016/j.ocemod.2007.07.001 
ARDHUIN F. et al. (2010). Semi-empirical dissipation source functions for ocean waves, part I: Definition, calibration and validation. Journal of Physical Oceanography, Vol. 40, pp 1917-1941. http://dx.doi.org/10.1175/2010JPO4324.1

BARAILLE R., FILATOFF N. (1995). Modèle shallow-water multicouches isopycnal de Miami. Rapport Technique 003/95, SHOM/CMO.

BERTIN X., BRUNEAU N., BREILH J.-N., FORTUNATO A.B., KARPYTCHEV M. (2012). Importance of wave age and resonance in storm surges : The case of Xynthia, bay of biscay. Ocean Modelling, Vol. 42, pp 16-30. http://dx.doi.org/10.1016/j.ocemod.2011.11.001

BISCARA L., SCHMITT T., CORREARD S., CREACH R. (2014). Modèles numériques de bathymétrie pour la prévision hydrodynamique du dispositif vigilance vagues-submersions. JNGCGC 2014, Dunkerque. http://dx.doi.org/10.5150/jngcgc.2014.060

BLECK R. (2002). An oceanic general circulation model framed in hybrid isopycnicCartesian coordinates. Ocean Modelling, Vol. 4(1), pp 55-88. http://dx.doi.org/10.1016/S14635003(01)00012-9

BOUDIERE E., MAISONDIEU C., ARDHUIN F., ACCENSI M., PINEAUGUILLOU L., LEPESQUEUR J. (2013). A suitable metocean hindcast database for the design of Marine energy converters. International Journal of Marine Energy, Vol. 3-4, pp e40-e52. http://dx.doi.org/10.1016/j.ijome.2013.11.010

CHARNOCK H. (1955). Wind Stress on a Water Surface. Quart. J. Roy. Meteorol. Soc., Vol. 81, pp 639-640. http://dx.doi.org/10.1002/qj.49708135027

IDIER D., KRIEN Y., PEDREROS R., DESRAMAUT N., PINEAU-GUILLOU L., DUMAS F. (2012). Système de prévision de surcotes en Manche/Atlantique et Méditerranée : Amélioration du système existant sur la façade Manche/Gascogne [d4]. Rapport Technique BRGM/RP-61019-FR, BRGM/IFREMER.

LEFEVRE J.M et al. (2009). Apport d'un nouveau modèle de vagues de $3^{\circ}$ génération à Météo France. Actes de conférence des Ateliers de Modélisation de l'Atmosphère, 2729 janvier 2009, Toulouse, France.

MICHAUD H., MARSALEIX P., LEREDDE Y., ESTOURNEL C., BOURRIN F., LYARD F., MAYET C., ARDHUIN F. (2012). Three-dimensional modelling of waveinduced current from the surf zone to the inner shelf. Ocean Science Vol. 8, pp 657-681. http://dx.doi.org/10.5194/os-8-657-2012

MAKIN V. (2005). A note on the drag of the sea surface at hurricane winds. BoundaryLayer Meteorology, Vol. 115(1), pp 169-176. http://dx.doi.org/10.1007/s10546-004-3647-x

PARADIS D. et al. (2013). Inter-comparaison de 3 modèles de surcotes dans le cadre du projet Homonim. Rapport Technique Météo-France/Dprévi

TOLMAN H. (2008). A mosaic approach to wind wave modelling. Ocean Modelling, Vol 25(1-2), pp 35-47.

WU J. (1982). Wind-stress coefficients over sea-surface from breeze to hurricane. Journal of geophysical research, Vol. 87(C12). pp 9704-9706 http://dx.doi.org/10.1029/JC087iC12p09704 